\title{
Physical Fitness Characterization by Obesity Level in Young Males with Poor Physical Fitness
}

\author{
Tamotsu Kitabayashi ${ }^{1}$, Shin-ichi Demura ${ }^{2}$, Takanori Noguchi ${ }^{3}$ \\ ${ }^{1}$ Tokyo University of Science, Tokyo, Japan \\ ${ }^{2}$ Graduate School of Natural Science \& Technology, Kanazawa University, Kanazawa, Japan \\ ${ }^{3}$ Fukui University of Technology, Fukui, Japan \\ Email: t-noguchi@fukui-ut.ac.jp
}

Received January $17^{\text {th }}$, 2013; revised February $19^{\text {th }}, 2013$; accepted March $4^{\text {th }}, 2013$

\begin{abstract}
Copyright (c) 2013 Tamotsu Kitabayashi et al. This is an open access article distributed under the Creative Commons Attribution License, which permits unrestricted use, distribution, and reproduction in any medium, provided the original work is properly cited.
\end{abstract}

\begin{abstract}
Lack of physical fitness in obese people, recent decline in physical fitness of young people, and physical fitness problems of thin people are a concern. We investigated the physical fitness characteristics of different obese groups comprising young males with poor physical fitness. Technical college students (144 males; age, $15.9 \pm 1.1 \mathrm{y}$; height, $169.1 \pm 5.9 \mathrm{~cm}$; weight, $60.0 \pm 13.3 \mathrm{~kg}$ ) took the physical fitness test of the Ministry of Education, Culture, Sports, Science and Technology (MEXT) and were judged to have lower than normal physical fitness in a comprehensive evaluation of the MEXT physical test. Three groups with different obesity levels were formed according to their percent body fat (\%BF): thin $(<15 \%)$, normal $(15 \%-25 \%)$, and obese $(>25 \%)$. The obese group had stronger grip than the thin and normal groups but was inferior to the thin group in 20-m shuttle run, standing broad jump, $50-\mathrm{m}$ run, and total score. The test requiring large physical movements revealed that the obese group was inferior in power and endurance. Therefore, among young males with poor physical fitness, obese individuals have different physical fitness characteristics than thin and normal individuals; they are inferior to normal and thin individuals in running- and jumping-related power and endurance but superior in muscle strength.
\end{abstract}

Keywords: Obesity Level; Physical Fitness; Exercise Prescription

\section{Introduction}

A marked decline in physical fitness of young people has been noted as a problem for more than $20 \mathrm{y}$. At the time, many studies were conducted on the decline in physical fitness due to physical inactivity, adiposity with an increased percent body fat (\%BF), and the occurrence of lifestyle-related diseases (Beunen, Malina, Ostyn, Renson, Simons, \& Van Gerven, 1983; Kim, Matsuura, Tanaka, \& Inagaki, 1993). In particular, much attention has been paid to the decline in physical fitness due to obesity. However, the causes for this decline have diversified and the number of young people with poor physical fitness and obese individuals are increasing. There has also been an increase in extremely thin young males (Hayano, 2002). A low $\% \mathrm{BF}$ in young people was not previously considered a problem because many of them exercised daily through club activities and had superior physical fitness.

However, the number of thin individuals with poor physical fitness has increased recently, despite them having a low \%BF similar to athletes. Thus, there is a need to distinguish thin people with good physical fitness from those with poor fitness. Hayano (2002) reported that thin individuals with poor physical fitness suffer from eating disorders because of their desire to lose excess weight or because of following incorrect diets (Hayano, 2002). As a result, there is a decrease in their muscle mass, which is necessary for physical activities. Physical fitness level generally reaches a peak between childhood and adoles- cence (Tokyo Metropolitan University, 2007). Therefore, increasing fitness levels sufficiently during this period is very important because it largely affects their physical fitness levels in adolescence (Barnekow, Hedberg, Janlert, \& Jansson, 1988; Iwai, Matuki, Koshida, Tanaka, Miyashita, \& Urabe, 2008; Mikkelsson, Kaprio, Kautiainen, Kujala, Mikkelsson, \& Nupponen, 2006). Therefore, physical fitness must be improved with adequate exercise prescriptions. However, obese and/or thin individuals are among those with poor physical fitness, and physical fitness characteristics may differ with the degrees of obesity. This study may be considered more significant than previous studies because it clarifies the causes of low physical fitness levels according to the degree of obesity.

We clarified the physical fitness characteristics in groups of young males with poor physical fitness and different degree of obesity.

\section{Method}

\section{Subjects}

The physical fitness test of the Ministry of Education, Culture, Sports, Science and Technology (MEXT) sets a judgment criterion for a comprehensive physical fitness based on the total score in each test (Five stages; A [highest] to E [lowest]). We targeted young males with poor physical fitness. Students corresponding to the $\mathrm{D}$ and $\mathrm{E}$ levels, which are lower than the 
standard level (C), were selected as subjects according to the general MEXT judgment criteria. In short, among 606 male technical college students from the first to third grade, 144 students (age, $15.9 \pm 1.1 \mathrm{y}$; height, $169.1 \pm 5.9 \mathrm{~cm}$; weight, 60.0 $\pm 13.3 \mathrm{~kg}$ ) were judged to have poor physical fitness (D and $\mathrm{E}$ levels).

The purpose and procedure of the study were explained in detail, and informed consent was obtained from all participants. This experimental protocol was approved by the Ethics Committee on Human Experimentation of Faculty of Education, Kanazawa University (authorization number: 19-4).

\section{\%BF and Body Mass Index (BMI)}

Subcutaneous fat thickness was measured at the triceps and scapular sites with a skinfold caliper (Cambridge Scientific Industries, Inc., Cambridge, MD, USA). After calculating body density by substituting the total value of subcutaneous fat thickness in the equation of Nagamine \& Suzuki (1964), we calculated \%BF using the equation of Brozek et al. (1951). BMI, which is an obesity index, was calculated by dividing the weight in $\mathrm{kg}$ by the height in $\mathrm{m} 2$ (Katherine, Margaret, Brian, \& Cynthia, 2012).

\section{Physical Fitness Test}

The MEXT physical fitness test was selected as the physical fitness test in this study (The Ministry of Education, Culture, Sports, Science and Technology, 2000). It consists of eight tests: grip strength (strength), sit-ups (muscle endurance), sit and reach (flexibility), side-stepping (agility), 20-m shuttle run (general endurance), 50-m run, standing broad jump, and handball throwing (muscle power; running, jumping, and throwing ability). The tests were performed according to the enforcement method for physical fitness tests (Sports and Youth Bureau of the MEXT, 2012: The Ministry of Education, Culture, Sports, Science and Technology, 2000).

Based on the score table provided by MEXT, the records of each test were converted into a scale of $0-10$, and the total scores were used as the general physical fitness evaluation value. The measurement of skinfold thickness and the physical fitness test were carried out from early April to early June during regular health and physical education classes held by physical education teachers with expertise and experience.

\section{Statistical Analysis}

The relationship between \%BF and BMI was examined using Pearson's correlation coefficient. A one-way analysis of variance (ANOVA, the obesity factor) was used to determine the mean differences for each physical test and total scores. When a significant main effect was found, a multiple comparison test was conducted using Tukey's honestly significant difference (HSD) method. The mean difference was assessed by effect size (ES); $p<.05$ was considered significant.

\section{Results}

The correlation between \%BF and BMI was significant and very high $(\mathrm{r}=.86)$. Based on $\% \mathrm{BF}$, three groups with different obesity levels were created: thin (<15\%BF), normal (15 - 25 $\% \mathrm{BF}$ ), and obese (>25\%BF). As a result, 73 (height, $168.7 \pm$ $5.5 \mathrm{~cm}$; weight, $52.8 \pm 6.1 \mathrm{~kg}$; \%BF, $12.3 \% \pm 2.1 \%$ ), 45 (height, $168.7 \pm 5.9 \mathrm{~cm}$; weight, $59.1 \pm 8.1 \mathrm{~kg} ; \% \mathrm{BF}, 18.3 \% \pm 1.9 \%)$, and 26 (height, $171.3 \pm 6.5 \mathrm{~cm}$; weight, $81.7 \pm 12.1 \mathrm{~kg} ; \% \mathrm{BF}$, $31.8 \% \pm 6.1 \%$ ) subjects were assigned to the thin, normal, and obese groups, respectively.

Table 1 shows the means and standard deviations of the physical fitness test scores based on the groups, the test results of the one-way ANOVA (degree of obesity), and ES. Significant main effects were obtained in the tests of grip strength, 20-m shuttle run, 50-m run, and standing broad jump. The thin and normal groups had lower total scores and grip strength but significantly higher 20 -m shuttle run and standing broad jump scores than the obese group. The thin group was faster in the 50-m run but had lower total scores than the obese group.

Table 1.

Physical characteristics and one-way ANOVA (Obesity levels).

\begin{tabular}{|c|c|c|c|c|c|c|c|c|c|c|c|}
\hline Fitness test & & Thin & $N=72$ & Normal & $\mathrm{N}=45$ & Obese & $\mathrm{N}=26$ & & & & \\
\hline & Unit & Mean & SD & Mean & SD & Mean & SD & F-value & $p$ & Tukey’s HSD & Effect Size \\
\hline Age & (Year) & 15.6 & 0.86 & 16.0 & 1.13 & 16.5 & 1.14 & & & & \\
\hline Height & $(\mathrm{cm})$ & 168.7 & 5.53 & 168.7 & 5.92 & 168.7 & 5.53 & & & & \\
\hline Weight & $(\mathrm{kg})$ & 52.8 & 6.06 & 59.1 & 8.14 & 81.7 & 12.14 & & & & \\
\hline BMI & & 18.5 & 1.71 & 20.7 & 2.49 & 27.9 & 4.45 & & & & \\
\hline Grip strength & $(\mathrm{kg})$ & 34.8 & 5.60 & 35.7 & 5.23 & 42.0 & 5.93 & $16.29^{*}$ & 0.00 & Thin, Nomal < Obese & Thin < Obese: 1.25 \\
\hline Sit-up & (Times) & 24.0 & 4.17 & 23.6 & 4.83 & 23.5 & 3.13 & 0.22 & 0.80 & & \\
\hline Sit and reach & (Times) & 39.6 & 6.28 & 39.8 & 9.72 & 42.5 & 7.56 & 1.39 & 0.25 & & \\
\hline Side-stepping & (Times) & 51.1 & 4.93 & 50.2 & 4.15 & 49.0 & 6.29 & 1.80 & 0.17 & & \\
\hline 20-m shuttle run & (Times) & 68.4 & 12.67 & 65.7 & 11.49 & 46.5 & 11.78 & $32.14^{*}$ & 0.00 & Obese $<$ Thin, Nomal & Obese < Thin: 1.76 \\
\hline 50-m run & (s) & 8.3 & 0.55 & 8.5 & 0.57 & 8.7 & 0.54 & $5.99^{*}$ & 0.00 & Thin $<$ Obese & Thin < Obese: .79 \\
\hline Standing broad jump & $(\mathrm{cm})$ & 200.5 & 17.78 & 192.9 & 14.86 & 180.7 & 23.10 & $11.87^{*}$ & 0.00 & Obese $<$ Thin, Nomal & Obese < Thin: 1.03 \\
\hline Handball throwing & $(\mathrm{m})$ & 18.0 & 3.28 & 18.1 & 4.16 & 17.1 & 4.05 & 0.68 & 0.51 & & \\
\hline Total score & (Points) & 37.9 & 4.33 & 36.9 & 4.98 & 35.2 & 4.99 & $3.46^{*}$ & 0.03 & Obese $<$ Thin & Obese < Thin: .61 \\
\hline
\end{tabular}

${ }^{*}: p<.05$. 
ESs between the mean of the obese group and the thin and normal groups were 1.24 and 1.14 for grip strength, 1.76 and 1.66 for the 20-m shuttle run, and 1.03 and .67 for the standing broad jump, respectively, and ES between the mean of the thin group and obesity group was .79 for the $50-\mathrm{m}$ run; the total score was .61.

\section{Discussion}

The MEXT physical fitness test classifies the physical fitness of young people into five grades based on the total score. The subjects in the present study were all judged to have poor physical fitness, corresponding to the D or E level, which is lower than the standard level (C) among the five grades. Hence, their physical fitness was considered to be inferior to that of young people of the same generation.

The degree of obesity is generally determined by \%BF. BMI calculated from the height and weight has also been used worldwide as a simple index of the degree of obesity in young people. However, BMI has a limitation when used for subjects with large muscle mass, such as athletes because of an over-evaluating effect (Demura, 2011). However, it may be a useful parameter to evaluate body composition in subjects other than athletes because a strong relationship exists between the two parameters $(r=.86)$; thus, the grouping based on \%BF were judged to be valid.

Handgrip strength is used as an index of muscle strength because the results can be generalized to the whole body (Demura, 2011; The Ministry of Education, Culture, Sports, Science and Technology, 2000). Grip strength was significantly greater in the obese group (42 kg) than in the normal and thin groups, with a large difference (ES $=1.14-1.24)$, and was comparable to that of the Technical college students (grade 1, $41.5 \mathrm{~kg}$; grade 2, $43.6 \mathrm{~kg}$; grade 3, $44.1 \mathrm{~kg}$ ) who engage in high frequency physical exercise (3 - 4 times/week) (Shimada, Demura, \& Yamada, 2010). On the other hand, grip strength of the thin group $(34.8 \mathrm{~kg})$ and the normal group $(35.7 \mathrm{~kg})$ was lower than that of the obese group by 7 - $8 \mathrm{~kg}$ on an average. Grip strength in the thin and normal groups was lower than the standard value of the same generation. Hence, inferior muscle strength may be a main factor of inferior physical fitness in the thin and normal groups.

In particular, thin individuals tended to enforce weight loss and dietary limitations more than that required because of an excessive desire for a slim body (Hayano, 2002). As a result, a risk of reduction in the amount of $\mathrm{BF}$ as well as muscle mass has been indicated.

The 20-m shuttle run has been used as an index of general endurance because of its strong correlation with $\mathrm{VO}_{2}$ max It was significantly inferior in the obese group than in the thin and normal groups (46.5 times), showing a very large difference $(\mathrm{ES}=1.66$ - 1.76). In contrast to muscle strength, general endurance in the obese group was particularly inferior compared with that in young people with poor physical fitness. In case of the 20-m shuttle run, heavy weight because of large body movements was considered to greatly affect performance and impose a large burden on the knee joints of obese subjects. A similar tendency was found in the 50-m run and standing broad jump that require rapid body movements, wherein the obese group was found to be inferior to the other groups.

The weight of obese subjects affects the motor ability tests, which involve large body movements such as running and jumping. Thus, it is necessary for obese subjects to reduce BF mass while maintaining muscle mass to improve these physical fitness elements related to running and jumping. No significant difference was observed for tests such as sit-ups (general endurance), sit and reach (flexibility), side-stepping (agility), and the handball throw (muscle power; throwing ability). These tests do not require much body movement and are completed within a relatively short time. Therefore, there was little effect of body weight on performance. However, the mean values for each group on these tests were equal to or less than the standard value. This may be the main cause of poor physical fitness among our subjects, which is common in all subjects with poor physical fitness.

In particular, scores of the handball throw test were lower than those of the other tests (approximately $18 \mathrm{~m}: 3$ points). The throwing movement is complex and requires energy generated by lower limbs to be amplified and transmitted smoothly from the trunk to the upper limbs. It also requires whole-body coordination in addition to muscle strength.

The following may be offered as the standard exercise prescription to improve physical fitness of young males with poor physical fitness. Strength improvement training is recommended for thin or normal people with poor physical fitness because they generally tend to have inferior muscular function. On the other hand, obese people need to improve endurance power through running/jumping exercises. These exercises may be combined with strength training to increase basal metabolism and aerobic exercise to reduce BF, considering the burden on blood pressure and the joints. Furthermore, it may be desirable to include ball games promoting nerve functions into an exercise program because all young people with poor physical fitness perform inadequate exercise.

\section{Conclusion}

In conclusion, the cause of poor physical fitness was different between the obese and thin groups among young people with poor physical fitness. The obese group was superior in muscle strength but inferior in general endurance and power related to running and jumping, whereas the thin group was inferior in muscle strength. Other physical fitness elements showed little group differences.

Among young males with poor physical fitness, obese individuals have different physical fitness characteristics from normal and thin individuals and are inferior to normal and thin individuals in power and endurance of the whole body related to running and jumping but superior in muscle strength.

\section{Acknowledgements}

This work was supported by Fukui National College Technology. The authors thank Prof. Shigeru Shimada and Dr. Takayoshi Yamada for their assistance with data collection. The authors also thank Enago (www.enago.jp) for the English language review.

\section{REFERENCES}

Barnekow-Bergkvist, M., Hedberg, G., Janlert, U., \& Jansson, E. (1988). Prediction of physical fitness and physical activity level in adulthood by physical performance and physical activity in adolescence-an 18-year follow-up study. Scandinavian Journal of Medicine 
\& Science in Sports, 8, 299-308. doi:10.1111/j.1600-0838.1998.tb00486.x

Beunen, G., Malina, M. R., Ostyn, M., Renson, R., Simons, J., \& Van Gerven, D. (1983). Fatness, growth and motor fitness of Belgian boys 12 through 20 years of age. Human Biology, 55, 599-613.

Brozek, J., \& Keys, A. (1951). The evaluation of leanness-fatness in man: Norms and interrelationships. British Journal of Nutrition, 5, 194-206. doi:10.1079/BJN19510025

Demura, S. (2011). Health and a sports science lecture (2nd ed., pp. 38-59). Tokyo: Kyorinsyoin Press.

Hayano, H. (2002). A psychological study on eating disorders tendency of male college students. Journal of Japanese Clinical Psychology, 20, 44-51.

Iwai, K., Matuki, M., Koshida, S., Tanaka, K., Miyashita, K., \& Urabe, Y. (2008). Association between progression of underweight and overweight status and physical fitness improvements in the youth: A one-year follow-up study. The Japanese Society of Physical Fitness and Sport Medicine, 57, 491-502. doi:10.7600/jspfsm.57.491

Katherine, F. M., Margaret, C. D., Brian, K. K., \& Cynthia, O. L. (2012). Prevalence of obesity and trends in the distribution of body mass index among US adults, 1999-2010. JAMA, 307, 491-497. doi:10.1001/jama.2012.39
Kim, R. H., Matsuura, Y., Tanaka, K., \& Inagaki, A. (1933). Physical fitness and motor ability in obese girls aged 12 to 14 years. The Japanese Society of Physical Fitness and Sport Medicine, 42, 380388.

Mikkelsson, L., Kaprio, J., Kautiainen, H., Kujala, U., Mikkelsson, M., \& Nupponen, H. (2006). School fitness tests as predictors of adult health-related fitness. American Journal of Human Biology, 18, 342349. doi:10.1002/ajhb.20498

Nagamine, S., \& Suzuki, S. (1964). Anthropometry and body composition of Japanese among men and women. Human Biology, 36, 8-15.

Shimada, S., Demura S., \& Yamada T. (2010). Characteristics of life style and health-status based on longitudinal data for three years of Japanese male students in the national college of technology. The Journal of Education and Health Science, 55, 293-304.

Sports and Youth Bureau of the MEXT (2012). The research report of physical fitness and exercise capacity 2011. Tokyo: The Ministry of Education, Culture, Sports, Science and Technology Press.

The Ministry of Education, Culture, Sports, Science and Technology (2000). New physical fitness test. Tokyo: Gyousei.

Tokyo Metropolitan University (2007). New standard value of Japanese physical fitness 2 . Tokyo: Fumaidou. 